\title{
A NOTION OF ANALYTIC GENERATOR FOR GROUPS OF UNBOUNDED OPERATORS
}

\author{
JOSÉ E. GALÉ \\ Departamento de Matemáticas, Universidad de Zaragoza \\ 50009 Zaragoza, Spain \\ E-mail: gale@unizar.es
}

Dedicated to Professor W. Żelazko on the occasion of his 70th birthday

\begin{abstract}
We introduce a notion of analytic generator for groups of unbounded operators, on Banach modules, arising from Esterle's quasimultiplier theory. Characterizations of analytic generators are given in terms of the existence of certain functional calculi. This extends recent results about $C_{0}$ groups of bounded operators. The theory is applicable to sectorial operators, representations of $H^{\infty}$, and integrated groups.
\end{abstract}

1. Introduction. Groups of unbounded operators (on Banach spaces) are important objects of research which require careful definition or treatment. They have been approached directly, see $[\mathrm{Hu}]$ for instance, or indirectly, in connection with well-behaved families of bounded operators such as $C$-regularized groups, integrated groups, or distribution groups $[\mathrm{dL}]$. On the other hand, it is a usual method to associate a single object or operator to families of (bounded or not) operators, in order to facilitate the study of such families. This is the case for $C_{0}$ groups of bounded operators, when one considers their infinitesimal generators, or alternatively their analytic generators [CZ]. Notions of infinitesimal generators have been given, and studied in some extent, for groups of unbounded operators (or for the aforementioned associated families) as well. Here, we are interested in analytic generators, and deal with groups of regular quasimultipliers of Banach algebras and Banach modules (see definitions below).

The theory of the analytic generator, say $U$, of a group of bounded operators on a

2000 Mathematics Subject Classification: Primary 47D03, 47A60, 47B48.

Key words and phrases: Banach algebras, multipliers, quasimultipliers, unbounded operators, analytic generator, functional calculus, sectorial operators, integrated groups.

Research of the author supported by Project BFM2001-1793 of the M.C.YT.D.G.I. and F.E.D.E.R, Spain.

The paper is in final form and no version of it will be published elsewhere. 
Banach space $X$ is well established, see [CZ], [M], [CK], [Mo] and references therein. Recently, it has been observed that there always exists a functional calculus $\Psi_{U}$ for such an operator $U$ which is unique, subject to certain assumptions. The operating functions are analytic on sectors which are centered at the origin and contain the spectrum of $U$. Moreover, these functions $f(\lambda)$ are allowed to grow polynomially in $\lambda$ as $\lambda \rightarrow \infty$, and polynomially in $\lambda^{-1}$ as $\lambda \rightarrow 0$, through the sectors, and therefore they include in particular all $H^{\infty}$ functions. This functional calculus produces closed operators $\Psi_{U}(f)$ on $X$ which are generally unbounded even if $f$ is an $H^{\infty}$ function [U].

Groups of regular quasimultipliers, which we denote generically by $\left(T_{t}\right)_{t \in \mathbb{R}}$, have been introduced in [GM]. Such a class of groups (of closed, unbounded operators) is quite general. It includes $C$-regularized groups, integrated groups, distribution groups, and, in particular, (formal) groups of the form $\left(e^{-i t \mathcal{L}}\right)_{t \in \mathbb{R}}$ arising from boundary values of holomorphic semigroups, or from solutions of ill-posed Cauchy problems [dL], [GM] (here, $\mathcal{L}$ denotes a sub-Laplacian operator on a Lie group, for example). Some applications to the above families are given in $[\mathrm{GM}]$, where the theory is presented in terms of certain canonical morphisms called Weyl homomorphisms (see definition below). In the present paper, it is shown that the usage of Weyl homomorphisms also enables us to define an explicit notion of analytic generator, say $H$, for a group $\left(T_{t}\right)_{t \in \mathbb{R}}$, which neatly extends the original concept for $C_{0}$ groups of bounded operators. We focus on possible relationships between analytic generators and functional calculi. In Theorem 2 below, a characterization of the analytic generator $H$ is given via the existence of a canonical functional calculus $\Psi$ for $H$, which is expressed as a certain representation between algebras of quasimultipliers.

The work done in [U] was motivated by the theory of sectorial operators and those of them having bounded imaginary powers (BIP operators, for short). More precisely, the calculus $\Psi_{U}$ of [U] generalizes the functional calculus associated with BIP operators. There has been recent interest in analyzing sectorial and/or BIP operators in connection with $H^{\infty}$ calculus, and analytic generators [BC], [CDMY], [Mo], [U]. In Theorem 4 we prove that all these concepts are essentially equivalent when they are placed in the wider setting of the quasimultiplier theory.

Theorem 2 and Theorem 4 extend results of $[\mathrm{U}]$ and $[\mathrm{CDMY}]$. In particular, $\Psi_{U}$ can be regarded as a particular case of representation in the well structured framework of Esterle's quasimultiplier theory. This view may be helpful to shed some light about the character of the resulting operators, as well as to shed some light on the fact that $H^{\infty}$ functions yield operators which are unbounded in general.

The paper is divided into three sections besides this introduction. In Section 1, we summarize basic features about the quasimultiplier theory, and about functional calculi for sectorial operators and $C_{0}$ groups of bounded operators. Section 3 contains Theorem 2 and Theorem 4. In the final section we illustrate our theorems with some examples including sectorial operators and integrated groups.

Some of the material in this paper is contained (although without much detail) in [GM]. To be more precise, the study of groups of regular quasimultipliers carried out in $[\mathrm{GM}]$ emphasizes aspects of their theory which mainly concern what we may call the "infinitesimal generator" of the group. The spirit of the present paper concerns the notion of analytic generator, whose definition as a quasimultiplier is given explicitly here. 
Acknowledgements. The author wishes to thank the referee for valuable comments and suggestions, which have improved considerably the presentation of this paper.

2. Preliminaries. 1) Quasimultipliers. Let $A$ be a complex commutative Banach algebra. A bounded linear operator $T: A \rightarrow A$ is said to be a multiplier of $A$ if $T(a b)=a T(b)$ for every $a, b \in A$. The set $\operatorname{Mul}(A)$ of all multipliers of $A$ is a unital and commutative Banach algebra with respect to the operator composition and norm, and it is usually called the multiplier algebra of $A$. If $L_{a}(x):=a x$, for $x, a \in A$, the canonical inclusion $a \mapsto L_{a}, A \subset \operatorname{Mul}(A)$ is always continuous. Now assume that the above algebra $A$ is such that $A^{\perp}=(0)$ and that $\Delta(A) \neq \emptyset$, where $A^{\perp}:=\{a \in A: a A=(0)\}$ and $\Delta(A):=\{a \in A: \overline{a A}=A\}$. Given $a \in A$ and $b \in \Delta(A)$, let $D_{a, b}=\{x \in A: a x \in b A\}$. Define the linear map $T_{a / b}: D_{a, b} \rightarrow A$ by setting $T_{a / b}(x)$ to be the unique element of $A$ such that $a x=b T_{a / b}(x)$. A mapping $T_{a / b}$ of this form is called a quasimultiplier on $A$. Clearly different choices of $a$ and $b$ can give rise to the same quasimultiplier. Every quasimultiplier is densely defined and closed on $A$, but quasimultipliers are not necessarily bounded. Let $Q M(A)$ denote the set of quasimultipliers on $A$. If $A$ is unital then $Q M(A)$ is isomorphic to $A$. In general $Q M(A)$ is isomorphic to the algebra of fractions $A / \Delta(A)$ and, in fact, it becomes a commutative convex bornological algebra with respect to a natural family of bounded subsets of $A$, which are called pseudobounded sets. A subset $\mathcal{U}$ of $Q M(A)$ is pseudobounded if there exists $u \in\left(\cap_{T \in \mathcal{U}} D(T)\right) \cap \Delta(A)$ for which $\sup _{T \in \mathcal{U}}\|T u\|<\infty$ (here $D(T)$ is $D_{a, b}$ if $T=T_{a / b}$, with $a \in A, b \in \Delta(A)$ ). Then $T \in Q M(A)$ is said to be a regular quasimultiplier provided that there exists $\lambda>0$ such that the subset $\left\{\lambda^{n} T^{n}: n=1,2, \ldots\right\}$ is pseudobounded. The subalgebra of $Q M(A)$ of all regular quasimultipliers on $A$ will be denoted by $Q M_{r}(A)$. It contains the multiplier algebra $\operatorname{Mul}(A)$ in such a way that bounded subsets of $\operatorname{Mul}(A)$ are pseudobounded in $Q M_{r}(A)$.

The algebra $Q M_{r}(A)$ admits a representation as an inductive limit of multiplier algebras, which relies upon the concept of similarity. Two Banach algebras $A$ and $B$ are called similar if there is another Banach algebra $I$ with $\Delta(I) \neq \emptyset$, which is continuously included (via injective homomorphisms) as dense ideals in $A$ and $B$. Similarity is an equivalence relation in the class of commutative Banach algebras with dense principal ideals, and two such Banach algebras $A, B$ satisfying $A^{\perp}=(0), B^{\perp}=(0)$ are similar if and only if $Q M(A)$ and $Q M(B)$, and then $Q M_{r}(A)$ and $Q M_{r}(B)$, are bornologically isomorphic (that is, there is an algebraic bijection between $Q M(A)$ and $Q M(B)$ which is also a bijection between the collection of pseudobounded subsets of $Q M(A)$ and those of $Q M(B)$ ). Let $\mathcal{S}(A)$ denote the set of all algebras in $Q M_{r}(A)$ which are similar to $A$. For $B_{1}, B_{2} \in \mathcal{S}(A)$ write $B_{1} \leq B_{2}$ if $B_{1} \subset B_{2}$ and $\operatorname{Mul}\left(B_{1}\right) \subset \operatorname{Mul}\left(B_{2}\right)$ (with the respective inclusion maps being norm-decreasing). The system $\{M u l(B)\}_{B \in \mathcal{S}(A)}$ is inductive, and we get that $Q M_{r}(A)$ is isomorphic to $\lim _{B \in \mathcal{S}(A)} M u l(B)$. This identification provides a correspondence between pseudobounded subsets of $Q M_{r}(A)$ and bounded subsets of $\operatorname{Mul}(B)$, for $B$ running over $\mathcal{S}(A)$. Namely, suppose that $\mathcal{U}$ is a pseudobounded subset of $Q M_{r}(A)$ stable under products. Then $\mathcal{I}_{\mathcal{U}}:=\left\{u \in \cap_{T \in \mathcal{U}} D(T): \sup _{T \in \mathcal{U}}\|T u\|<\infty\right\}$ is a dense ideal in $A$ which becomes a Banach algebra in its own right, when endowed with the norm $p(u):=\max \left(\sup _{T \in \mathcal{U}}\|T u\|,\|u\|\right),\left(u \in \mathcal{I}_{\mathcal{U}}\right)$. The norm $p$ satisfies $\|u\| \leq p(u)$, 
$p(a u) \leq\|a\| p(u), p(T u) \leq p(u)$ for every $u \in \mathcal{I}_{\mathcal{U}}, a \in A, T \in \mathcal{U}$. We take $B_{\mathcal{U}}$ as the completion of $A$ in the multiplier algebra $\operatorname{Mul}\left(\mathcal{I}_{\mathcal{U}}\right)$. Then $\mathcal{U}$ is contained in the closed unit ball of $\operatorname{Mul}\left(B_{\mathcal{U}}\right)$ and $\operatorname{Mul}(A) \hookrightarrow \operatorname{Mul}\left(B_{\mathcal{U}}\right)$.

An important tool in the treatment of quasimultipliers is given by the following fact. Let $A$ and $B$ be two Banach algebras such that $A^{\perp}=B^{\perp}=(0)$ and $\Delta(A), \Delta(B) \neq \emptyset$, and let $\varphi: A \rightarrow B$ a bounded algebra homomorphism such that either $\varphi(A) B$ or $\varphi(A)$ is dense in $B$. Then the correspondence $a^{\prime} / a \mapsto \varphi\left(a^{\prime}\right) / \varphi(a)$, where $a^{\prime} \in A, a \in \Delta(A)$, defines a pseudobounded (extension of $\varphi$ ) morphism from $Q M(A)$ into $Q M(B)$, and from $Q M_{r}(A)$ into $Q M_{r}(B)$.

For the above, and other facts concerning the quasimultiplier theory, we refer the reader to $[\mathrm{E}]$.

2) Groups of quasimultipliers. Let $\omega$ be a continuous weight on $\mathbb{R}$ and let $L^{1}(\omega)$ be the Beurling algebra corresponding to $\omega$. We have that $L^{1}(\omega)^{\perp}=(0)$ and $\Delta\left(L^{1}(\omega)\right) \neq \emptyset$ and so the algebras $Q M_{r}\left(L^{1}(\omega)\right)$ and $Q M\left(L^{1}(\omega)\right)$ exist. To see that $\Delta\left(L^{1}(\omega)\right) \neq \emptyset$ note that the set of functions $g^{\lambda, z}(r):=\frac{1}{\sqrt{4 \pi \lambda}} \exp \left[-(r+i z)^{2} /(4 \lambda)\right]\left(r \in \mathbb{R}, \lambda \in \mathbb{C}^{+}, z \in \mathbb{C}\right)$, defines a holomorphic biparameter semigroup $g^{\lambda, z}$ in $L^{1}(\omega)$ such that $g^{\lambda, z} \in \Delta\left(L^{1}(\omega)\right)$ for every $\lambda \in \mathbb{C}^{+}$and $z \in \mathbb{C}\left(\mathbb{C}^{+}\right.$denotes here the right hand half-plane of complex numbers $)$. Note that $g^{\lambda}:=g^{\lambda, 0}$ is the Gaussian semigroup on $\mathbb{R}$ and that $\left(g^{\lambda}\right)_{0<\lambda<1}$ is a bounded approximate identity for $L^{1}(\omega)$.

In [GM], an $\omega$-group in $Q M_{r}(A)$ is defined as a family $\left(T_{t}\right)_{t \in \mathbb{R}}$ of regular quasimultipliers of $A$ such that $T_{0}$ is the identity operator $I d$ and

(i) there exists $u \in \bigcap_{s, t \in \mathbb{R}} D\left(T_{s} T_{t}\right) \cap \Delta(A)$ such that

(a) the map $t \mapsto T_{t} u$ is continuous, and

(b) for all $s, t \in \mathbb{R}, T_{s}\left(T_{t} u\right)=T_{s+t}(u)$,

(ii) $\left(\omega(t)^{-1} T_{t}\right)_{t \in \mathbb{R}}$ is pseudobounded in $Q M_{r}(A)$.

Let $\mathcal{U}:=\left\{\lambda T_{t} \in Q M_{r}(A): t \in \mathbb{R}, \lambda \in \mathbb{C},|\lambda| \leq \omega(t)^{-1}\right\}$. Then $\mathcal{U}$ is pseudobounded and stable under products. The ideal $\mathcal{I}:=\mathcal{I}_{\mathcal{U}}$ defined by $\mathcal{U}$ as indicated above will be called the $\omega$-ideal of $A$ associated to $T_{t}$. As shown before, there exists a Banach algebra $B:=B_{\mathcal{U}}$ similar to $A$ such that $\left(T_{t}\right)_{t \in \mathbb{R}}$ is contained in the unit ball of $M u l(B)$. In fact, $\left(T_{t}\right)_{t \in \mathbb{R}}$ becomes a strongly continuous $C_{0}$ group of multipliers of $B$ satisfying $\left\|T_{t}\right\|_{M u l(B)}=O(\omega(t))$, as $|t| \rightarrow \infty$. The algebra $B$ is called the $\omega$-algebra of $A$. The mapping $\Theta: L^{1}(\omega) \rightarrow M u l(B)$ defined by $\Theta(f) \xi=\int_{-\infty}^{\infty} f(t) T_{t} \xi d t\left(\xi \in B, f \in L^{1}(\omega)\right)$, is a bounded algebra homomorphism which extends to a pseudobounded homomorphism between the respective algebras of quasimultipliers $Q M\left(L^{1}(\omega)\right) \stackrel{\Theta}{\longrightarrow} Q M(A), Q M_{r}\left(L^{1}(\omega)\right) \stackrel{\Theta}{\longrightarrow} Q M_{r}(A)$, given by $\Theta(f / g):=\Theta(f) u^{2} / \Theta(g) u^{2}$ where $u \in \mathcal{I} \cap \Delta(A)$ and $f / g \in Q M\left(L^{1}(\omega)\right)$. The mapping $\Theta$ is called the Weyl homomorphism of $\left(T_{t}\right)_{t \in \mathbb{R}}$.

Under suitable conditions, the scope of the above constructions can be widened to operators on Banach spaces. Let $X$ be a Banach space and let $A$ be a Banach algebra, with $\Delta(A) \neq \emptyset$, for which $X$ is a left Banach $A$-module such that $\overline{A X}=X$ and $X_{A}^{\perp}=(0)$, where $X_{A}^{\perp}:=\{a \in A: a x=0$ for all $x \in X\}$. Note that $\overline{A X}=X$ and $X_{A}^{\perp}=(0)$ imply that $A^{\perp}=(0)$. To transfer quasimultipliers of $A$ into (unbounded) operators on $X$, put $D\left(T_{X}\right)=\{x \in X:(T a) x \in a X\}$ and define $T_{X}: D\left(T_{X}\right) \rightarrow X$ by $T_{X}(x)=y$, if 
$x \in D\left(T_{X}\right)$, where $y$ is the only element in $X$ such that $(T a) x=a y$ (the uniqueness of $y$ follows from the fact that $\left.A_{X}^{\perp}=(0)\right)$. It is readily seen that $D(T) X \subset D\left(T_{X}\right), D\left(T_{X}\right)$ is dense in $X$, and $T_{X}$ is closed on $X$. Then we define $Q M(X ; A):=\left\{T_{X}: T \in Q M(A)\right\}$ and $Q M_{r}(X ; A):=\left\{T_{X}: T \in Q M_{r}(A)\right\}$.

For $w$-groups and the above observations we refer the reader to [GM].

3) Functional calculi. Let $\tau$ be such that $0<\tau<\pi$ and put $S_{\tau}=\{\lambda \in \mathbb{C} \backslash\{0\}$ : $|\arg (\lambda)|<\tau\}$, where $\arg (\lambda)$ is the principal branch of the argument taking values in $[-\pi, \pi)$. Let $H^{\infty}\left(S_{\tau}\right)$ be the usual Banach algebra of bounded analytic functions on $S_{\tau}$. By $\mathcal{A}_{b}\left(S_{\tau}\right)$ we denote the Banach subalgebra of $H^{\infty}\left(S_{\tau}\right)$ containing all functions in $H^{\infty}\left(S_{\tau}\right)$ which admit bounded, continuous extensions to $\overline{S_{\tau}} \backslash\{0\}$. Set $\psi(\lambda):=\lambda(1+\lambda)^{-2}$, if $\lambda \in S_{\tau}$. For $\delta \geq 0$, we define $\mathcal{A}_{0}^{\delta}\left(S_{\tau}\right)$ as the subalgebra of all functions $f$ of $\mathcal{A}_{b}\left(S_{\tau}\right)$ for which $f(\lambda) \psi^{-\delta}(\lambda) \rightarrow 0$ as $|\lambda| \rightarrow \infty$ or $|\lambda| \rightarrow 0$. Put $\mathcal{D} \mathcal{R}\left(S_{\tau}\right):=\bigcup_{\delta>0} \mathcal{A}_{0}^{\delta}\left(S_{\tau}\right)$ and $\mathcal{F}\left(S_{\tau}\right):=\bigcup_{\delta>0} \psi^{-\delta} H^{\infty}\left(S_{\tau}\right) \equiv \bigcup_{\delta>0} \psi^{-\delta} \mathcal{A}_{b}\left(S_{\tau}\right)$. Clearly, $\mathcal{D} \mathcal{R}\left(S_{\tau}\right) \subset H^{\infty}\left(S_{\tau}\right) \subset \mathcal{F}\left(S_{\tau}\right)$.

A closed operator $H$ on a Banach space $X$ is said to be sectorial if it is one-to-one, with dense domain and range in $X$, with spectrum $\sigma(H)$ contained in $\overline{S_{\rho}}$ (for some $\rho \geq 0$ ), and such that, for every $\tau>\rho,\left\|(z-H)^{-1}\right\| \leq C_{\tau}|z|^{-1}$ whenever $z \in \mathbb{C} \backslash S_{\tau}$. Take the (positively oriented) path $\gamma(t):=|t| e^{-\operatorname{sign}(t) \cdot \tau}(t \in \mathbb{R})$. Then the Dunford-Riesz integral $\frac{1}{2 \pi i} \int_{\gamma}(z-H)^{-1} h(z) d z$ is well defined for every $h \in \mathcal{D} \mathcal{R}\left(S_{\tau}\right)$, it does not depend on $\tau>\rho$ and yields an algebra homomorphism $\Upsilon: \mathcal{D} \mathcal{R}\left(S_{\tau}\right) \rightarrow \mathcal{B}(X)$, where $\mathcal{B}(X)$ denotes the usual Banach algebra of bounded operators on $X$. Moreover, if $f \in \mathcal{F}\left(S_{\tau}\right)$ then $f \psi^{k} \in \mathcal{D} \mathcal{R}\left(S_{\tau}\right)$ for some integer $k \geq 0$. So the operator $\Upsilon(f):=\Upsilon\left(\psi^{k}\right)^{-1} \Upsilon\left(\psi^{k} f\right)$, which does not depend on $k$, is a closed operator on $X$ (note that $\Upsilon\left(\psi^{k}\right)$ is injective). The mapping $f \mapsto \Upsilon(f)$ defines a functional calculus for $H$ whose properties are explained in [CDMY]. For a sectorial operator $H$, the imaginary powers $H^{i s}, s \in \mathbb{R}$, always exist as closed operators on $X$. When $H^{i s}$ is bounded, for every $s \in \mathbb{R}, H$ is said to be a BIP operator [CDMY].

Suppose that $\left(T_{s}\right)_{s \in \mathbb{R}}$ is a $C_{0}$ group in $\mathcal{B}(X)$ with analytic generator $U$ [CZ], [Mo]. Let $\mathcal{M}$ denote the Mellin transform defined by $\mathcal{M}(h)(s)=\frac{1}{2 \pi} \int_{0}^{\infty} h(\lambda) \lambda^{i s-1} d \lambda, s \in \mathbb{R}$, for every $h \in \mathcal{D} \mathcal{R}\left(S_{\tau}\right)$. Using the residue theorem we get $|\mathcal{M}(h)(s)| \leq C_{\delta, \tau} e^{-\tau|s|}(s \in \mathbb{R})$. Hence, $\mathcal{M}(h)$ belongs to $L^{1}\left(\omega_{\rho}\right)$, where $\sigma<\rho<\tau$ and $\omega_{\rho}(s):=\exp (\rho|s|)(s \in \mathbb{R})$. Put $\tilde{\mathcal{M}}(h)(s):=\mathcal{M}(h)(-s), s \in \mathbb{R}, h \in \mathcal{D} \mathcal{R}\left(S_{\tau}\right)$. Let $\Theta$ be the Weyl homomorphism corresponding to $\left(T_{s}\right)_{s \in \mathbb{R}}$ in this case, i. e., $f \mapsto \int_{-\infty}^{\infty} f(s) T_{s} d s, L^{1}\left(\omega_{\rho}\right) \rightarrow \mathcal{B}(X)$. Then $\Psi_{U}:=\Theta \circ \tilde{\mathcal{M}}$ is a functional calculus on $\mathcal{D} \mathcal{R}\left(S_{\tau}\right)$ which can be extended by approximation to $\mathcal{F}\left(S_{\tau}\right)$. This extended calculus gives rise to closed, not necessarily bounded, operators on $X$. The properties of $\Psi_{U}$ have been studied in [U]. In the particular case where $T_{s}=H^{i s}, s \in \mathbb{R}$, for a BIP operator $H$, we have that $\Psi_{U}=\Upsilon[\mathrm{U}]$. Thus the calculus $\Psi_{U}$ generalizes the calculus for sectorial operators.

3. Analytic generators of $\omega$-groups and functional calculus. In all of this section, $X$ is assumed to be a Banach space and a Banach left module on a Banach algebra $A$ as at the end of Section 2. Let $\omega$ be a continuous weight on $\mathbb{R}$ and let $\left(T_{t}\right)_{t \in \mathbb{R}}$ be an $\omega$-group of regular quasimultipliers of $A$ with Weyl homomorphism $\Theta$. Let $\mathcal{I}$ denote the $\omega$-ideal of $A$ associated to $T_{t}$. If $u$ is in $\Delta(A) \cap \mathcal{I}$ then $\Theta\left(g^{1}\right) u^{2}$ generates a dense ideal of $A$. For 
every $z \in \mathbb{C}$, the quasimultiplier of $A$ defined as $\frac{\Theta\left(g^{1, z}\right) u^{2}}{\Theta\left(g^{1}\right) u^{2}}$ does not depend on $u$. We will denote it by $\frac{\Theta\left(g^{1, z}\right)}{\Theta\left(g^{1}\right)}$.

Definition 1. Put $H_{z}:=\frac{\Theta\left(g^{1, z}\right)}{\Theta\left(g^{1}\right)}, z \in \mathbb{C}$. The family $\left(H_{z}\right)_{z \in \mathbb{C}}$ of quasimultipliers of $A$ is called the complex quasimultiplier extension of $\left(T_{t}\right)_{t \in \mathbb{R}}$. We define the analytic generator of $\left(T_{t}\right)_{t \in \mathbb{R}}$ to be the quasimultiplier $H_{1}$.

As in Section 2, we may extend these definitions to the case where $\left(T_{t}\right)_{t \in \mathbb{R}}$ is an $\omega$-group of regular quasimultipliers on an $A$-module $X$.

REMARK 1. The above definition is consistent with the classical one of the analytic generator of a group of bounded operators. To see this, suppose for a moment that the group $\left(T_{t}\right)_{t \in \mathbb{R}}$ is a $C_{0}$ group in $\mathcal{B}(X)$ (of growth $\omega$ at infinity). For $z \in \mathbb{C}$ such that $\Re z \neq 0$, let $\Sigma_{z}$ denote the strip $\{\lambda \in \mathbb{C}: 0<\Re \lambda / \Re z<1\}$. Recall that the analytic continuation of $T_{t}$ is the family of (generally unbounded) closed operators $\left(U_{z}, D\left(U_{z}\right)\right)_{z \in \mathbb{C}}$ on $X$ defined as follows. If $\Re z \neq 0$, the domain $D\left(U_{z}\right)$ of $U_{z}$ is the subset of $x \in X$ for which there is a $X$-valued function $F_{x}(\lambda)$, holomorphic in $\Sigma_{z}$ and continuous in $\bar{\Sigma}_{z}$, such that $F_{x}(i s)=T_{s}$ if $s \in \mathbb{R}$. Then $U_{z}(x):=F_{x}(z)$ for every $x \in D\left(U_{z}\right)$. If $z=i s, s \in \mathbb{R}$, then $D\left(U_{i s}\right):=X$ and $U_{i s} x:=T_{s} x$ for every $x \in X$. The Cioranescu-Zsido's analytic generator, for $\left(T_{t}\right)_{t \in \mathbb{R}}$, is defined to be the operator $U:=U_{1}[\mathrm{CZ}]$, [Mo].

Now, let $\Theta$ be the Weyl homomorphism of $T_{t}$. The closed subalgebra $A$ of $\mathcal{B}(X)$ generated by $\Theta\left(L^{1}(\omega)\right)$ satisfies $A^{\perp}=(0)$ and $\Delta(A) \neq \emptyset$. For each $z \in \mathbb{C}$, set $H_{z}$ in $Q M(A)$ as in Definition 1. Take $x$ in $D\left(U_{z}\right)$ and $y \in X$ such that $U_{z} x=y \in X$. If $F_{x}$ is the holomorphic function on $\bar{\Sigma}_{z}$ as above, we have $\Theta\left(g^{1}\right) F_{x}(i s)=\Theta\left(g^{1}\right) T_{s} x=\Theta\left(g^{1} * \delta_{s}\right) x=$ $\Theta\left(g^{1, i s}\right) x$ for all $s \in \mathbb{R}$. By the uniqueness principle, $\Theta\left(g^{1}\right) F_{x}(\lambda)=\Theta\left(g^{1, \lambda}\right) x$ on $\bar{\Sigma}_{z}$. In particular, $\Theta\left(g^{1}\right) y=\Theta\left(g^{1}\right) F_{x}(z)=\Theta\left(g^{1, z}\right) x$ and then it follows that $x \in D\left(H_{z}\right)$ and $H_{z} x=y$. Hence $U_{z} \subset H_{z}$. Conversely, take $x$ in $D\left(H_{z}\right) \subset X$ and let $y$ be in $X$ such that $\Theta\left(g^{1, z}\right) x=\Theta\left(g^{1}\right) y$. For $n \in \mathbb{N}$, put $x_{n}=\Theta\left(g^{\frac{1}{n}}\right) x$. Using again analyticity and the fact that $\Theta\left(g^{\frac{1}{n}, i s}\right) x=\Theta\left(\delta_{s}\right) \Theta\left(g^{\frac{1}{n}}\right) x=T_{s} x_{n}(s \in \mathbb{R})$, we obtain that $x_{n} \in D\left(U_{z}\right)$ with $U_{z} x_{n}=$ $\Theta\left(g^{\frac{1}{n}, z}\right) x$. Moreover, $\lim _{n \rightarrow \infty} x_{n}=x$ and $U_{z} x_{n}=\Theta\left(g^{\frac{1}{n}}\right) \frac{\Theta\left(g^{1, z}\right)}{\Theta\left(g^{1}\right)} x=\Theta\left(g^{\frac{1}{n}}\right) y \rightarrow_{n \rightarrow \infty} y$. Since $\left(U_{z}, D\left(U_{z}\right)\right)$ is closed, we have that $x \in D\left(U_{z}\right)$ and $U_{z} x=y$. Thus we have seen that $H_{z} \subset U_{z}$. In conclusion, $H_{z}=U_{z}$.

REMARK 2. Let $B$ be the $\omega$-algebra of $A$ corresponding to the $\omega$-group $T_{t}$. Then $\left(T_{t}\right)_{t \in \mathbb{R}}$ is a $C_{0}$ group in $\operatorname{Mul}(B)$ and we can apply Remark 1. So, if $U_{B, z}$ is the analytic continuation of $\left(T_{t}\right)_{t \in \mathbb{R}}$ on $B$, we have that $U_{B, z}=\frac{\Theta\left(g^{1, z}\right)}{\Theta\left(g^{1}\right)}$, for $z \in \mathbb{C}$. Hence $H_{1}=U_{B, 1}$ on $B$.

REmark 3. The family $W(z):=\Theta\left(g^{1, i z}\right)$ is the entire $C$-group considered in [CK, p. 3589] for $C=\Theta\left(g^{1}\right)$, see also [dL]. In this respect, the representation $C^{-1} W(-i)$ of the analytic generator which is obtained in [CK, Theorem 2.6] corresponds formally to that of $H_{1}$ given in Definition 1. For relationships between groups of regular quasimultipliers and $C$-groups, see [GM].

Next, we will give a theorem about functional calculus which is a generalization of theorems in [U]. Let $\tau>0$ and $\delta \geq 0$. It is readily seen that the algebra $\mathcal{A}_{0}^{\delta}\left(S_{\tau}\right)$ defined in 
Section 1, part 3), is a Banach algebra, and a Banach $\mathcal{A}_{b}\left(S_{\tau}\right)$-module, when endowed with the norm $\|f\|_{\delta, \infty}:=\left\|f \psi^{-\delta}\right\|_{\infty}\left(f \in \mathcal{A}_{0}^{\delta}\left(S_{\tau}\right)\right)$. Note that $\mathcal{A}_{0}^{\delta}\left(S_{\tau}\right)$ is an integral domain. Put $a^{z}(\lambda):=\exp \left[-z(\log \lambda)^{2}\right]$, where $\log \lambda=\log |\lambda|+i \arg (\lambda)$, if $\lambda \in S_{\tau}$ and $z \in \mathbb{C}^{+}$. Then $\left(a^{z}\right)_{\Re z>0}$ is an analytic semigroup in $\mathcal{A}_{0}^{\delta}\left(S_{\tau}\right)$ such that $\overline{a^{z} \mathcal{A}_{0}^{\delta}\left(S_{\tau}\right)}=\mathcal{A}_{0}^{\delta}\left(S_{\tau}\right)$ for each $z \in \mathbb{C}^{+}$. From the above we get $\Delta\left(\mathcal{A}_{0}^{\delta}\left(S_{\tau}\right)\right) \neq \emptyset$ and $\mathcal{A}_{0}^{\delta}\left(S_{\tau}\right)^{\perp}=(0)$, and so the algebras $Q M_{r}\left(\mathcal{A}_{0}^{\delta}\left(S_{\tau}\right)\right)$ and $Q M\left(\mathcal{A}_{0}^{\delta}\left(S_{\tau}\right)\right)$ exist. Indeed, the algebras $\mathcal{A}_{0}^{\delta}\left(S_{\tau}\right)$ and $\mathcal{A}_{0}^{\delta^{\prime}}\left(S_{\tau}\right)$ are similar for every $\delta, \delta^{\prime} \geq 0$, and it can be proved that $Q M_{r}\left(\mathcal{A}_{0}^{\delta}\left(\Sigma_{\tau}\right)\right)=\operatorname{Mul}\left(\mathcal{A}_{0}^{\delta}\left(\Sigma_{\tau}\right)\right)=$ $\mathcal{A}_{b}\left(S_{\tau}\right)$, for every $\delta \geq 0$. Moreover, $\psi^{k} \in \mathcal{A}_{0}^{\delta}\left(\Sigma_{\tau}\right)$ for every $k \geq \delta$. Also, $\psi^{-k} a^{1}$ is in $\mathcal{A}_{0}^{\delta}\left(\Sigma_{\tau}\right)$ and so $a^{1} \mathcal{A}_{0}^{\delta}\left(\Sigma_{\tau}\right) \subset \psi^{k} \mathcal{A}_{0}^{\delta}\left(\Sigma_{\tau}\right)$.

This implies that $\psi^{k} \in \Delta\left(\mathcal{A}_{0}^{\delta}\left(\Sigma_{\tau}\right)\right)$. As every function $f$ in $\mathcal{F}\left(S_{\tau}\right)$ can be expressed by $f=\left(f \psi^{k}\right) / \psi^{k}$ for $k>0$ big enough, we obtain that $\mathcal{F}\left(S_{\tau}\right) \subset Q M\left(\mathcal{A}_{0}^{\delta}\left(\Sigma_{\tau}\right)\right)$. For the above results, see $[\mathrm{GM}]$.

If $\tau>0$ we put $\omega_{\tau}(s):=\exp (\tau|s|)(s \in \mathbb{R})$. For $w \in \mathbb{C}$, let $\zeta_{w}$ denote the function $\zeta_{w}(\lambda)=\lambda^{w} \equiv e^{w \log \lambda}, \lambda \in \mathbb{C} \backslash(-\infty, 0]$.

Theorem 2. Let $A$ be a Banach algebra such that $\Delta(A) \neq \emptyset$ and let $X$ be a left Banach $A$-module such that $X_{A}^{\perp}=(0)$ and $\overline{A X}=X$. Assume that $H$ is a closed, densely defined operator on $X$. Fix $\sigma \in[0, \pi)$. The following assertions are equivalent.

(1) There exists a family $\left(T_{t}\right)_{t \in \mathbb{R}}$ in $Q M_{r}(A)$, which is an $\omega_{\tau}$-group for every $\tau \in(\sigma, \pi)$, whose analytic generator is $H$.

(2) There exists a unital, pseudobounded homomorphism

$$
\Psi: Q M\left(\mathcal{A}_{0}^{\delta}\left(S_{\tau}\right)\right) \rightarrow Q M(A),
$$

independent of $\delta>0$ and $\tau \in(\sigma, \pi)$, with $\Psi\left(\Delta\left(\mathcal{A}_{0}^{\delta}\left(S_{\tau}\right)\right)\right) \Delta(A) \cap \Delta(A) \neq \emptyset$, such that $\Psi\left(\zeta_{1}\right)=H$.

(3) There exists a unital, pseudobounded homomorphism

$$
\Psi: \mathcal{A}_{b}\left(S_{\tau}\right) \rightarrow Q M_{r}(A),
$$

independent of $\tau \in(\sigma, \pi)$, with $\Psi\left(\Delta\left(\mathcal{A}_{0}^{\delta}\left(S_{\tau}\right)\right)\right) \Delta(A) \cap \Delta(A) \neq \emptyset$ for every $\delta>0$, such that $\Psi\left(\zeta_{1} a^{1}\right)=\Psi\left(a^{1}\right) H$ in $Q M_{r}(A)$.

Proof. (1) implies (2). The argument is partly taken from [GM, Section 6]. Take $\delta>0$ and $\tau \in(\sigma, \pi)$. Let $\mathcal{M}$ denote the Mellin transform. As in Section 1, part 3$), \tilde{\mathcal{M}}$ is a well defined, bounded algebra homomorphism from $\mathcal{A}_{0}^{\delta}\left(S_{\tau}\right)$ into $L^{1}\left(\omega_{\rho}\right)$, whenever $\sigma<\rho<\tau$. It is straightforward to check that $\tilde{\mathcal{M}}\left(\mathcal{A}_{0}^{\delta}\left(S_{\tau}\right)\right)$ contains the ideal $g^{1} * C_{c}(\mathbb{R})$, whence it follows that $\tilde{\mathcal{M}}$ has dense range in $L^{1}\left(\omega_{\rho}\right)$. Thus $\tilde{\mathcal{M}}$ extends as a pseudobounded homomorphism $Q M\left(\mathcal{A}_{0}^{\delta}\left(S_{\tau}\right)\right) \stackrel{\tilde{\mathcal{M}}}{\longrightarrow} Q M\left(L^{1}\left(\omega_{\rho}\right)\right)$. Let $\Theta$ be the Weyl homomorphism of $\left(T_{t}\right)_{t \in \mathbb{R}}, \Theta: Q M_{r}\left(L^{1}\left(\omega_{\rho}\right)\right) \rightarrow Q M_{r}(A)$. Define $\Psi:=\Theta \circ \tilde{\mathcal{M}}$. Observe that $\tilde{\mathcal{M}}\left(\zeta_{1} a^{1}\right)(s)$ agrees with the inverse Fourier transform of the function $t \mapsto e^{-t^{2}} e^{-t}$ at $s$, that is, the function $g^{1,1}(s)$, for every $s \in \mathbb{R}$. Hence, $\Psi\left(\zeta_{1}\right)=\Psi\left(\zeta_{1} a^{1}\right) / \Psi\left(a^{1}\right)=\Theta\left(g^{1,1}\right) / \Theta\left(g^{1}\right)=$ $H_{1}=: H$. In analogous manner, $\Psi(1)=I d$. Finally, note that $\Psi\left(a^{1}\right) u^{2} \equiv \Theta\left(g^{1}\right) u^{2}$ generates a dense ideal in $A$ provided that $u$ in $\Delta(A)$.

(2) implies (3). Since $\Psi$ is pseudobounded, it sends regular quasimultipliers into regular quasimultipliers. Also, $\Psi\left(\zeta_{1} a^{1}\right)=\Psi\left(\zeta_{1}\right) \Psi\left(a^{1}\right)=\Psi\left(a^{1}\right) H$ in $Q M_{r}(A)$. 
(3) implies (1). Take $\tau \in(\sigma, \pi)$. Let $\Omega$ be the unit ball of $\mathcal{A}_{b}\left(S_{\tau}\right)$ and put $\mathcal{U}:=\Psi(\Omega)$. Then $\mathcal{U}$ is a, stable under products, pseudobounded subset of $Q M_{r}(A)$. Put $B:=B_{\mathcal{U}}$ where $B_{\mathcal{U}}$ is the Banach algebra, similar to $A$, obtained from $\mathcal{U}$ as in Section 1 . Since $\mathcal{U}$ is contained in the unit ball of $\operatorname{Mul}(B), \Psi$ goes continuously from $\mathcal{A}_{b}\left(S_{\tau}\right)$ into $\operatorname{Mul}(B)$. Now, note that $\left(\zeta_{i s}\right)_{s \in \mathbb{R}}$ is a $C_{0}$ group of operators in $\mathcal{A}_{b}\left(S_{\tau}\right)$ (acting by multiplication on $\mathcal{A}_{0}^{\delta}\left(S_{\tau}\right)$, where $\left.\delta>0\right)$ such that $\left\|\zeta_{i s}\right\|_{\infty} \leq M e^{\tau|s|}(s \in \mathbb{R})$. Define $T_{s}:=\Psi\left(\zeta_{i s}\right)$, if $s \in \mathbb{R}$. By the density condition assumed on $\Psi$ and the density of $A$ in $B$, it is readily seen that $T_{s}$ is a $C_{0}$ group in $\operatorname{Mul}(B)$ satisfying $\left\|T_{s}\right\| \leq M^{\prime} e^{\tau|s|}(s \in \mathbb{R})$. This means that $T_{s}$ is an $\omega_{\tau}$-group in $Q M_{r}(B)=Q M_{r}(A)$. Finally, let $\Theta$ be the Weyl homomorphism associated with $T_{s}$ and take $\rho \in(\tau, \pi)$. If $h \in \mathcal{A}_{0}^{\delta}\left(S_{\rho}\right)$ then $f:=\tilde{\mathcal{M}}(h)$ lies in $L^{1}\left(\omega_{\tau}\right)$. For $\varphi \in \Delta\left(\mathcal{A}_{0}^{\delta}\left(S_{\tau}\right)\right)$ and $u \in \Delta(A)$ such that $\Psi(\varphi) u \in \Delta(A)$, we obtain

$$
\Theta(f) \Psi(\varphi) u=\int_{-\infty}^{\infty} f(s) \Psi\left(\zeta_{i s} \varphi\right) u d s=\Psi\left(\int_{-\infty}^{\infty} f(s) \zeta_{i s} \varphi d s\right) u=\Psi(h \varphi) u=\Psi(h) \Psi(\varphi) u
$$

in $\operatorname{Mul}(B)$, by the continuity of $\Psi$ and the inversion of the Mellin transform. So $\Psi=$ $\Theta \circ \tilde{\mathcal{M}}$. In particular, $\Theta\left(g^{1,1}\right) / \Theta\left(g^{1}\right)=\Theta \tilde{\mathcal{M}}\left(\zeta_{1} a^{1}\right) / \Theta \tilde{\mathcal{M}}\left(a^{1}\right)=\Psi\left(\zeta_{1} a^{1}\right) / \Psi\left(a^{1}\right)=H$ as we wanted to show.

REMARK. The above theorem gives us the canonical functional calculus for analytic generators of $\omega$-groups (of regular quasimultipliers). In the proof, we have obtained the equalities $\Psi=\Theta \circ \tilde{\mathcal{M}}$ and $T_{s}=\Psi\left(\zeta_{i s}\right), s \in \mathbb{R}$, which readily induce a one-to-one correspondence between groups $\left(T_{s}\right)_{s \in \mathbb{R}}$ and calculi $\Psi$. Note also that part 3$)$ of the theorem is a result about $H^{\infty}$ representations (since $H^{\infty}\left(S_{\rho}\right) \subset \mathcal{A}_{b}\left(S_{\tau}\right)$ if $\rho>\tau$ ). In the special case of $C_{0}$ groups of bounded operators, the calculus $\Psi$ is strongly related to sectorial operators, bounded imaginary powers, $H^{\infty}$ representations [U], [CDMY]. There exist counterexamples which show that these concepts or properties do not coincide generally $[\mathrm{BC}],[\mathrm{CDMY}],[\mathrm{U}],[\mathrm{Mo}]$. Nevertheless, we will next show that all such notions are esentially equivalent in the quasimultiplier context.

The following is a feasible definition for sectorial quasimultipliers.

Definition 3. Let $A$ be a Banach algebra such that $A^{\perp}=(0)$ and $\Delta(A) \neq \emptyset$. A closed operator $H$ on $A$ is called a $Q M_{r}$-sectorial operator of angle $\sigma \geq 0$ if

(i) $H$ is one-to-one on $A$ and has domain and range dense in $A$.

(ii) For every $z \notin \bar{S}_{\sigma}$, there exists the inverse $(z-H)^{-1}$ of $z-H$ in $Q M(A)$ and, if $\tau \in(\sigma, \pi)$ then there is $M_{\tau}>0$ such that the set $\left\{M_{\tau}^{-1} z(z-H)^{-1}: z \notin \bar{S}_{\tau}\right\}$ is contained in a pseudobounded, stable under products, subset of $Q M_{r}(A)$.

Note that if $\mathcal{U}_{\tau}$ is a pseudobounded subset, stable under products, of $Q M_{r}(A)$ which contains $\left\{M_{\tau}^{-1} z(z-H)^{-1}: z \notin \bar{S}_{\tau}\right\}$, then $\mathcal{U}_{\tau}$ gives rise to a Banach algebra $B_{\tau}$, similar to $A$, such that $\left\{M_{\tau}^{-1} z(z-H)^{-1}: z \notin \bar{S}_{\tau}\right\}$ becomes included in the unit ball of $M u l\left(B_{\tau}\right)$. Thus the inverse $(z-H)^{-1}$ of $z-H$ lies in $\operatorname{Mul}\left(B_{\tau}\right) \subset \mathcal{B}\left(B_{\tau}\right)$, and so the spectrum of $H$ as (closed) operator on $B_{\tau}$ is contained in $\bar{S}_{\tau}$. Further, $\left\|(z-H)^{-1}\right\| \leq M_{\tau}|z|^{-1}$, for every $z \notin \bar{S}_{\tau}$. In other words, $H$ is sectorial on $B_{\tau}$, of angle $\tau$ at least. In particular, the imaginary powers $H^{i s}, s \in \mathbb{R}$, exist as closed operators on $B_{\tau}$ [Mo, p. 165]. 
TheOREM 4. Let $A$ be a Banach algebra such that $\Delta(A) \neq \emptyset$ and let $X$ be a left Banach $A$-module such that $X_{A}^{\perp}=(0)$ and $\overline{A X}=X$. Assume that $H$ is a closed, densely defined operator on $X$. Fix $\sigma \in[0, \pi)$. The following are equivalent.

(1) $H$ is the analytic generator of a group in $Q M_{r}(A)$ which is an $\omega_{\tau}$-group for every $\tau \in(\sigma, \pi)$.

(2) $H$ is $Q M_{r}$-sectorial in $A$ of angle $\sigma$.

(3) $H$ is $Q M_{r}$-sectorial in $A$ of angle $\sigma$ and $\left(H^{i s}\right)_{s \in \mathbb{R}}$ is an $\omega_{\tau}$-group in $Q M_{r}(A)$, for every $\tau \in(\sigma, \pi)$.

(4) $H$ is $Q M_{r}$-sectorial in $A$ of angle $\sigma$, and there exists a unital, pseudobounded homomorphism

$$
\Psi: H^{\infty}\left(S_{\tau}\right) \rightarrow Q M_{r}(A)
$$

independent of $\tau \in(\sigma, \pi)$, with $\Psi\left(\Delta\left(\mathcal{A}_{0}^{\delta}\left(S_{\tau}\right)\right)\right) \Delta(A) \cap \Delta(A) \neq \emptyset$, such that $\Psi\left(\zeta_{\text {is }}\right)=$ $H^{\text {is }}$ in $Q M_{r}(A)$, for every $s \in \mathbb{R}$.

Proof. (1) implies (2). Suppose that $H$ is the analytic generator of an $\omega_{\tau}$-group $T_{s}$, that is, $H=\Theta\left(g^{1,1}\right) / \Theta\left(g^{1}\right)$ where $\Theta$ is the Weyl morphism of $T_{s}$. We know that $H$, being a quasimultiplier, is densely defined on $A$. Let $\mathcal{I}, B$ be, respectively, the $\omega$-ideal and $\omega$ algebra of $A$ associated to $T_{s}$. Take $u \in \Delta(A) \cap \mathcal{I}$. From the general theory of $\omega$-groups, we have that $\left\{\Theta\left(g^{t}\right) u^{2} a: a \in A, t>0\right\}$ is dense in $A$ and that $M u l(B) \mathcal{I} \subset \mathcal{I}[\mathrm{GM}]$. Since $\Theta\left(g^{t}\right) u^{2}=H \Theta\left(g^{t,-1}\right) u^{2}$ and $\Theta\left(g^{t,-1}\right) u^{2} \in M u l(B) \mathcal{I} \subset \mathcal{I} \subset A$, it follows that the range of $H$ is dense in $A$. Now assume that $H a=0$ for some $a \in A$. Then $\Theta\left(g^{1,1}\right) a u^{2}=0$ and so we obtain by density that $a b=0$ for every $b \in A$, that is, $a \in A^{\perp}=(0)$. It follows that $H$ is injective on its domain in $A$.

Finally, let $\rho, \tau$ be such that $\sigma<\rho<\tau<\pi$, and take $\delta \geq 0$. For $z \notin \bar{S}_{\tau}$, put $r_{z}(\lambda):=(z-\lambda)^{-1}\left(\lambda \in S_{\rho}\right)$. Let $\Psi$ be the calculus given in Theorem 2. Clearly, $\left(z-\zeta_{1}\right) r_{z}=$ 1 in $Q M\left(\mathcal{A}_{0}^{\delta}\left(S_{\rho}\right)\right)$ and so $(z-H) \Psi\left(r_{z}\right)=\Psi\left(r_{z}\right)(z-H)=I d$ in $Q M(A)$. It follows that $\Psi\left(r_{z}\right)$ is the inverse $(z-H)^{-1}$ of $z-H$ in $Q M(A)$. Moreover, $r_{z} \in \mathcal{A}_{b}\left(S_{\rho}\right)$ and $\left\|r_{z}\right\|_{\infty} \leq C(\tau-\rho)^{-1}|z|^{-1}$ (where the sup- norm is taken on $S_{\rho}$ ) for some constant $C$. This means that, for some constant $M_{\rho, \tau}$, the subset $\left\{M_{\rho, \tau}^{-1} z r_{z}: z \notin \bar{S}_{\rho}\right\}$ is contained in the unit ball $\Omega$ of $\mathcal{A}_{b}\left(S_{\rho}\right)$ and therefore $\left\{M_{\rho, \tau}^{-1} z(z-H)^{-1}: z \notin \bar{S}_{\rho}\right\} \subset \Psi(\Omega)$, where $\Psi(\Omega)$ is stable under products and pseudobounded in $Q M_{r}(A)$.

Altogether, we have shown that $H$ is $Q M_{r}$-sectorial (of angle $\sigma$ ).

(2) implies (3). Let $\rho \in(\sigma, \pi)$. As we have seen prior to this theorem, $H$ is sectorial on the Banach space (algebra) $B_{\rho}$ (we maintain previous notations). Thus the the DunfordRiesz calculus $\Upsilon$ is defined from $\mathcal{A}_{0}^{\delta}\left(S_{\tau}\right)$ into $\operatorname{Mul}\left(B_{\rho}\right)$, if $\tau \in(\rho, \pi)$ and $\delta>0$. Put $\psi(\lambda)=\lambda(1+\lambda)^{-2}, \lambda \in \mathbb{C} \backslash\{-1\}$, as in Section 1 . For every $s \in \mathbb{R}$, we define $\Upsilon\left(\zeta_{\text {is }}\right):=$ $\Upsilon\left(\psi^{-k}\right) \Upsilon\left(\zeta_{i s} \psi^{k}\right)$ where $k$ is an integer bigger than $\delta$. Then $\Upsilon\left(\zeta_{i s}\right)=H^{i s}, s \in \mathbb{R}$, as closed operators on $B_{\rho}$ [U, p. 352]. On the other hand, $\Upsilon\left(\psi^{k}\right)$ has dense range in $B_{\rho}$ [CDMY, p. 55], and therefore $\Upsilon\left(\mathcal{A}_{0}^{\delta}\left(S_{\tau}\right)\right) B_{\rho}$ is dense in $B_{\rho}$. Then $\Upsilon$ extends to a pseudobounded homomorphism $\Psi: \mathcal{A}_{b}\left(S_{\tau}\right) \rightarrow Q M_{r}\left(B_{\rho}\right) \equiv Q M_{r}(A)$ given by $\Psi(f)=\Upsilon\left(f \psi^{k}\right) / \Upsilon\left(\psi^{k}\right)$ for each $f \in \mathcal{A}_{b}\left(S_{\tau}\right)$ (recall that $\psi^{k} \in \Delta\left(\mathcal{A}_{0}^{\delta}\left(S_{\tau}\right)\right.$ ), as noticed prior to Theorem 2) [GM]. In particular we get $\Psi\left(\zeta_{i s}\right)=\Upsilon\left(\zeta_{i s} \psi^{k}\right) / \Upsilon\left(\psi^{k}\right) \equiv \Upsilon\left(\zeta_{i s}\right)=H^{i s}$, if $s \in \mathbb{R}$, and then we obtain that $\left(H^{i s}\right)_{s \in \mathbb{R}}$ is an $\omega_{\tau}$-group in $Q M_{r}(A)$. 
(3) implies (4). Let $\Theta$ be the Weyl morphism associated with the $\omega$-group $H^{i s}$, and let $\Psi$ be the homomorphism $\Psi=\Theta \circ \tilde{\mathcal{M}}$ given in Theorem 2, part (3). Without loss of generality, we can consider $\Psi$ defined from $H^{\infty}\left(S_{\tau}\right)$ into $Q M_{r}(A)$. Further, $\Psi\left(\zeta_{i s}\right)=$ $\Psi\left(\zeta_{i s} a^{1}\right) / \Psi\left(a^{1}\right)$ with $\Psi\left(\zeta_{i s} a^{1}\right)=\Theta\left(g^{1} * \delta_{s}\right)$, whence $\Psi\left(\zeta_{i s}\right)=\Theta\left(\delta_{s}\right) \equiv H^{i s}$, for all $s \in \mathbb{R}$.

(4) implies (1). Take $T_{s}:=H^{i s}, s \in \mathbb{R}$. From the assumptions $T_{s}$ is an $\omega_{\tau}$-group in $Q M_{r}(A)$, if $\tau \in(\sigma, \pi)$. Let $B$ be a Banach algebra similar to $A$ such that $H$ is sectorial on $B$ and $T_{s}$ is a $C_{0}$ group in $\operatorname{Mul}(B)$. This means that $H$ is BIP on $B$ and therefore $H$ is the analytic generator of $H^{i s}$ on $B$ [Mo, p. 168]. In particular, if $\Theta$ is the Weyl morphism of $T_{s}$, then $H=\Theta\left(g^{1,1}\right) / \Theta\left(g^{1}\right)$ on $B$ (Remark 2). Viewing $H$ in this equality as a quasimultiplier, it follows that $H$ is the analytic generator of $T_{s}$ in $Q M(A)$.

Besides the relations $\Psi=\Theta \circ \tilde{\mathcal{M}}$ and $T_{s}=\Psi\left(\zeta_{i s}\right), s \in \mathbb{R}$, obtained in Theorem 2, we have found in Theorem 4 that $\left.\Psi\right|_{\mathcal{F}\left(S_{\tau}\right)}=\Upsilon$ and $T_{s}=H^{i s}, s \in \mathbb{R}$. From the last equality we get immediately the following corollary, which is to be compared with [U, p. Corollary 6.2] and [Mo, Proposition 3.14].

Corollary 5. Let $\left(T_{s}\right)_{s \in \mathbb{R}}$ and $\left(V_{s}\right)_{s \in \mathbb{R}}$ be two $\omega$-groups in $Q M_{r}(A)$ with the same analytic generator $H$. Then $T_{s}=V_{s}$ for every $s \in \mathbb{R}$.

\section{Some examples and applications}

(i) $C_{0}$-groups of bounded operators. Let $\left(T_{t}\right)_{t \in \mathbb{R}}$ be a $C_{0}$-group in $\mathcal{B}(X)$, with Weyl homomorphism $\Theta: L^{1}(\omega) \rightarrow \mathcal{B}(X)$. It has been observed in Remark 1 that the (classical) analytic generator of $T_{t}$ agrees with $\Theta\left(g^{1,1}\right) / \Theta\left(g^{1}\right)$ in $Q M(A)$ where $A$ is the Banach algebra $A=\overline{\Theta\left(L^{1}(\omega)\right)}$ in $\mathcal{B}(X)$. The functional calculus $\Psi_{U}$, for $\left(T_{t}\right)_{t \in \mathbb{R}}$, given in [U] can be now regarded as the restriction, to $\mathcal{F}\left(S_{\tau}\right)$, of the homomorphism $\Psi$ which appears in Theorem 2 and Theorem 4 above. So in particular we see that the unbounded, closed operators $\Psi_{U}(f), f \in \mathcal{F}\left(S_{\tau}\right)$, are quasimultipliers on $X$.

Let us remark that it is possible for $A$ to have a unit. Using $\Theta\left(g^{1 / n}\right), n \in \mathbb{N}$, as a bounded approximate identity in $A$, it is straightforward to check that $A$ has a unit if and only if $T_{t}$ is norm-continuous at the origin or, equivalently, that $t \mapsto T_{t}$ is an entire function from $\mathbb{C}$ into $A$.

(ii) Holomorphic semigroups of bounded operators. Let $\left(a^{z}\right)_{z>0}$ be a holomorphic semigroup in $\mathcal{B}(X)$, with $\overline{a^{z} X}=X\left(z \in \mathbb{C}^{+}\right)$, and such that $\left\|a^{1+i t}\right\|=O(\omega(t))$ as $|t| \rightarrow \infty$ where $\omega$ is a continuous weight on $\mathbb{R}$. Put $A:=\overline{\operatorname{span}}\left\{a^{z}: z \in \mathbb{C}^{+}\right\}$in $\mathcal{B}(X)$. It is readily seen that $A^{\perp}=(0)$. Also, $a^{z} \in \Delta(A)$ for every $z \in \mathbb{C}^{+}$. This is a consequence of the fact that $A=\overline{\cup_{r>0} a^{r} A}$ by definition, and that $\overline{\cup_{r>0} a^{r} A}=\overline{a^{z} A}$, for every $z \in \mathbb{C}^{+}$, by the uniqueness principle for holomorphic functions. So the algebras $Q M_{r}(A)$ and $Q M(A)$ exist. If we define the boundary values of $a^{z}$ by $T_{t}:=a^{1+i t} / a^{1}(t \in \mathbb{R})$, then $\left(T_{t}\right)_{t \in \mathbb{R}}$ is an $\omega$-group in $Q M_{r}(A)[\mathrm{GM}]$. Using the residue theorem we find that $\Theta\left(g^{1, z}\right)=\Theta\left(g^{1}\right) a^{z}$ for every $z \in \mathbb{C}^{+}$, where $\Theta$ is the Weyl homomorphism of $T_{t}$. Hence $a^{1}=\Theta\left(g^{1,1}\right) / \Theta\left(g^{1}\right)$ in $Q M(A)$, in fact in $A$. This means that $a^{1}$ is the analytic generator of $T_{t}$. Of course, this applies to $C_{0}$ groups on $i \mathbb{R}$ having analytic extension to the right hand half-plane. Compare with [Mo, Corollary 3.6]. 
(iii) Sectorial operators. Let $H$ be a sectorial operator on a Banach space $X$ of angle $\sigma, 0 \leq \sigma<\pi$. Take $\delta>0$ and $\tau \in(\sigma, \pi)$. Let $\Upsilon_{\delta}$ denote the restriction of the DunfordRiesz calculus for $H$ to the Banach algebra $\mathcal{A}_{0}^{\delta}\left(S_{\tau}\right)$. Put $A_{\delta}:=\overline{\Upsilon_{\delta}\left(\mathcal{A}_{0}^{\delta}\left(S_{\tau}\right)\right)}$ in $\mathcal{B}(X)$. We know that $\left\{\Upsilon_{\delta}(f) y: f \in \mathcal{A}_{0}^{\delta}\left(S_{\tau}\right), y \in X\right\}$ is dense in $X$, so that $c x=0$ for all $x \in X$, if $c \in A_{\delta}^{\perp}$. This implies that $c=0$ in $A_{\delta}$. Also, $A_{\delta}$ has dense principal ideals. By density, as usually, we get the extensions $\Upsilon_{\delta}: Q M\left(\mathcal{A}_{0}^{\delta}\left(S_{\tau}\right)\right) \rightarrow Q M\left(A_{\delta}\right) \equiv Q M\left(X ; A_{\delta}\right)$ and $\Upsilon_{\delta}: \mathcal{A}_{b}\left(S_{\tau}\right) \rightarrow Q M_{r}\left(A_{\delta}\right)$ of $\Upsilon_{\delta}$. As seen before, $\mathcal{F}\left(S_{\tau}\right)$ is (strictly) contained in $Q M\left(\mathcal{A}_{0}^{\delta}\left(S_{\tau}\right)\right)$ and, also, $\Upsilon_{\delta}$ equals both the representation $\Psi$ and the functional calculus $\Upsilon$. In this way, the calculus for sectorial operators appears as a "sub-calculus" of the quasimultiplier representation $\Psi$.

On the other hand, there are sectorial operators having bounded imaginary powers, that is, BIP operators, without $H^{\infty}$-calculus. In fact, the existence of $H^{\infty}$-calculus has been characterized in several ways, see $[\mathrm{BdL}],[\mathrm{CDMY}]$ for instance. According to Theorem 2, Theorem 4, and the observation in the last paragraph, the action of $H^{\infty}$ functions, in the functional calculus for BIP operators, yield naturally regular quasimultipliers, which in general are not bounded.

It may be worth noticing that the lack (in general) of the $H^{\infty}$-calculus $\Psi: \mathcal{A}_{b}\left(S_{\tau}\right) \rightarrow$ $\mathcal{B}(X)$ is a question on (the lack of) bounded approximate identities: If $\delta=0$, it happens that $\Psi: \mathcal{A}_{b}\left(S_{\tau}\right) \rightarrow \mathcal{B}(X)$ continuously if and only if $\Psi: \mathcal{A}_{0}^{0}\left(S_{\tau}\right) \rightarrow \mathcal{B}(X)$ continuously, because, $\left(a^{\lambda}\right)_{0<\lambda<1}$ being a bounded approximate identity in $\mathcal{A}_{0}^{0}\left(S_{\tau}\right)$, the homomorphism $\Psi$ extends, from $\mathcal{A}_{0}^{0}\left(S_{\tau}\right)$, to the algebra $\mathcal{A}_{b}\left(S_{\tau}\right)=\operatorname{Mul}\left(\mathcal{A}_{0}^{0}\left(S_{\tau}\right)\right)$ into $\mathcal{B}(X)$ [E, p. 96$]$. If $\delta>0$, we have that $\Psi$ is continuous from $\mathcal{A}_{0}^{\delta}\left(S_{\tau}\right)$ into $\mathcal{B}(X)$ too, but the algebra $\mathcal{A}_{0}^{\delta}\left(S_{\tau}\right)$ has no bounded approximate identities, and so the extension of $\Psi$ to $\mathcal{A}_{b}\left(S_{\tau}\right)$ is only possible via regular quasimultipliers.

Finally, note that it is possible for $A_{\delta}$ to have a unit. By applying part (i) of this section to the group $\left(H^{i s}\right)_{s \in \mathbb{R}}$, we obtain that $A_{\delta}$ possesses a unit if and only if $\sigma(H)$ is compact and does not contain the origin.

(iv) Integrated groups. Integrated groups and semigroups have been introduced in recent years, in connection with abstract Cauchy problems. They form currently an important, widely developed, area of research. For definitions and basic properties, the reader is referred to $[\mathrm{ABHN}],[\mathrm{H}],[\mathrm{deL}],[\mathrm{Mi}]$. Let $\alpha \geq 0$, and let $\left(T_{\alpha}(t)\right)_{T \in \mathbb{R}}$ be an $\alpha$-times integrated group in $\mathcal{B}(X)$ such that $\left\|T_{\alpha}(t)\right\| \leq C e^{k|t|}(t \in \mathbb{R})$, for some constants $C, k>0$. The generator $L$ of $T_{\alpha}(t)$ is defined by $(\lambda-L)^{-1} x=\lambda^{\alpha} \int_{0}^{\infty} e^{-\lambda t} T_{\alpha}(t) x d t(x \in X)$, where $\lambda>k$. The operator $L$ is closed and densely defined on $X$.

It has been shown in [Mi] that there exists a certain commutative, convolution Banach algebra $\mathcal{T}^{(\alpha)}$ on $\mathbb{R}$ such that the mapping $\mathcal{G}: f \mapsto \int_{-\infty}^{\infty} W_{0}^{\alpha} f(t) T_{\alpha}(t) d t, \mathcal{T}^{(\alpha)} \rightarrow \mathcal{B}(X)$ is a (well defined) bounded algebra homomorphism. (Here, $W_{0}^{\alpha} f$ denotes the bilateral Weyl fractional derivative of order $\alpha$, see [GM].) The algebra $\mathcal{T}^{(\alpha)}$ contains the space of test functions on $\mathbb{R}$ and then $\mathcal{G}\left(\delta^{\prime}\right)$ can be defined in a natural way, by approximation, as a closed, densely defined operator on $X$ (here, $\delta^{\prime}$ is the differentiation operator, which agrees with the operator defined by convolution with the distribution $\delta^{\prime} \equiv \delta_{0}^{\prime}$ ). In fact, $L=-\mathcal{G}\left(\delta^{\prime}\right)$ with the same domains [Mi]. 
Now, take $A_{\alpha}:=\overline{\mathcal{G}\left(\mathcal{T}^{(\alpha)}(\varphi)\right)}$ in $\mathcal{B}(X)$. Then $A_{\alpha}^{\perp}=(0)$ and $\Delta\left(A_{\alpha}\right) \neq \emptyset$, and $\mathcal{G}$ extends to a pseudobounded homomorphism between the corresponding algebras of quasimultipliers. Moreover, $\left(\delta_{s}\right)_{s \in \mathbb{R}} \subset Q M_{r}\left(\mathcal{T}^{(\alpha)}\right)$ and therefore $T_{s}:=\mathcal{G}\left(\delta_{s}\right), s \in \mathbb{R}$, is an $\omega$-group in $Q M_{r}\left(A_{\alpha}\right)$ for every weight $\omega \geq \varphi$. Also, the Weyl homomorphism $\Theta$ of $T_{s}$ factorizes as $\Theta: Q M_{r}\left(L^{1}(\omega)\right) \hookrightarrow Q M_{r}\left(\mathcal{T}^{(\alpha)}\right) \stackrel{\mathcal{G}}{\rightarrow} Q M_{r}\left(A_{\alpha}\right)[\mathrm{GM}]$.

Definition 6. We define the analytic generator of the $\alpha$-times integrated group $T_{\alpha}(t)$ to be the quasimultiplier $H$ of $A_{\alpha}$ given by $H=\Theta\left(g^{1,1}\right) / \Theta\left(g^{1}\right) \equiv \mathcal{G}\left(g^{1,1}\right) / \mathcal{G}\left(g^{1}\right)$.

If it were necessary, the domain of $H$ in $X$ could be limited to vectors $x \in D\left(H_{X}\right)$ for which $\frac{\Theta\left(g^{1, z}\right)}{\Theta\left(g^{1}\right)} x$ varies holomorphically in $z$.

The theory of the analytic generator of an integrated group may be developed along similar lines as those of [CZ], $[\mathrm{M}]$ and related papers. We finish this paper by showing that the natural relationship between generators $H$ and $L$ holds in $Q M\left(A_{\alpha}\right)$. Suppose that $T_{\alpha}(t)$ is an $\alpha$-times integrated group such that $\left\|T_{\alpha}(t)\right\| \leq K e^{\rho|t|},(t \in \mathbb{R})$, for some $\rho \in[0, \pi)$. Take $\tau \in(\rho, \pi)$. The function $f(\lambda)=\log (\lambda)\left(\lambda \in S_{\tau}\right)$ can be written as $f=f a^{1} / a^{1}$ and so it belongs to $Q M\left(\mathcal{A}_{0}^{\delta}\left(S_{\tau}\right)\right)$, for $\delta>0$. Applying the calculus $\Psi$ which is associated to the group $T_{t}=\mathcal{G}\left(\delta_{t}\right) \equiv \Theta\left(\delta_{t}\right)$ we obtain $\Psi\left(f a^{1}\right)=\Theta\left[\tilde{\mathcal{M}}\left(f a^{1}\right)\right]=$ $\Theta\left[i\left(g^{1}\right)^{\prime}\right]=i \Theta\left(\delta^{\prime} * g^{1}\right)$ and $\Psi\left(a^{1}\right)=\Theta\left(g^{1}\right)$ whence $\Psi(f)=i \Theta\left(\delta^{\prime}\right)=-i L$. In other words, $L=i \log H$ as quasimultipliers.

\section{References}

[ABHN] W. Arendt, Ch. J. K. Batty, M. Hieber and F. Neubrander, Vector-Valued Laplace Transforms and Cauchy Problems, Birkhäuser, Basel, 2001.

[BC] J. B. Baillon and Ph. Clément, Examples of unbounded imaginary powers of operators, J. Funct. Anal. 100 (1991), 419-434.

[BdL] K. N. Boyadzhiev and R. deLaubenfels, Semigroups and resolvents of bounded variation, imaginary powers and $H^{\infty}$ functional calculus, Semigroup Forum 45 (1992), 372-384.

[CK] I. Cioranescu and V. Keyantuo, Entire regularizations of strongly continuous groups and products of analytic generators, Proc. Amer. Math. Soc. 128 (1982), 3587-3593.

[CZ] I. Cioranescu and L. Zsido, Analytic generators for one-parameter groups, Tôhoku Math. J. 28 (1976), 327-362.

[CDMY] M. Cowling, I. Doust, A. McIntosh and A. Yagi, Banach operators with a bounded $H^{\infty}$ functional calculus, J. Austral. Math. Soc. 60 (1996), 51-89.

[dL] R. de Laubenfels, Existence Families, Functional Calculi and Evolution Equations, Lecture Notes in Math. 1570, Springer, Berlin, 1994.

[E] J. Esterle, Quasimultipliers, representations of $H^{\infty}$, and the closed ideal problem for commutative Banach algebras, in: Radical Banach Algebras and Automatic Continuity (Long Beach 1981), J. M. Bachar et al. (eds.), Lecture Notes in Math. 975, Springer, Berlin, 1983, 66-162.

[GM] J. E. Galé and P. J. Miana, One parameter groups of regular quasimultipliers, preprint, University of Zaragoza, 2004.

[H] M. Hieber, Laplace transforms and $\alpha$-times integrated semigroups, Forum Math. 3 (1991), 595-612. 
[Hu] R. J. Hughes, Semigroups of unbounded linear operators in Banach spaces, Trans. Amer. Math. Soc. 230 (1977), 113-145.

[M] E. Marschall, On the analytical generator of a group of operators, Indiana Univ. Math. J. 35 (1986), 289-309.

[Mi] P. J. Miana, Almost-distributions and integrated families, preprint, University of Zaragoza, 2003.

[Mo] S. Monniaux, A new approach to the Dore-Venni theorem, Math. Nachr. 204 (1999), 163-183.

[U] M. Uiterdijk, A functional calculus for analytic generators of $C_{0}$ groups, Int. Eq. Oper. Theory 36 (2000), 349-369. 